\title{
Clinico-Pathological Study of K-ras Mutations in Colorectal Tumors: A Single-Center Retrospective Study of 51 Patients in Madinah, Saudi Arabia
}

\author{
Nasser Mulla ${ }^{1}$, Abdulraheem Alshareef ${ }^{2}$, Abdul Rahman Syed ${ }^{3}$, Majid Al-Jahel ${ }^{4}$ \\ 1. Department of Internal Medicine, College of Medicine, Taibah University, Madinah, SAU 2. Department of Medical \\ Laboratories Technology, College of Medicine, Taibah University, Madinah, SAU 3. Department of Pathology, King \\ Fahad Hospital, Ministry of Health, Madinah, SAU 4. Department of Oncology, King Fahad Hospital, Ministry of Health, \\ Madinah, SAU
}

Corresponding author: Nasser Mulla,dr_nasser_m@hotmail.com

\section{Abstract \\ Background}

Colorectal cancer (CRC) is one of the leading types of cancer worldwide and in Saudi Arabia. At the molecular level, CRC is very complicated and requires establishing comprehensive patient stratification models through identification of patients who will benefit or will not benefit from targeted therapy. We retrospectively investigated and analyzed the frequency of Kirsten-ras (K-ras) mutation and its correlation with patients' characteristics as weel as its association with clinicopathological features (i.e age, gender, clinical stage, anatomical site, histological subtype, degree of histological differentiation and metastatic site) in patients with CRC.

\section{Methods}

Medical records and paraffin-embedded tumor samples from 51 patients with histologically proven colorectal adenocarcinoma referred to Madinah center in Saudi Arabia were analyzed for the occurrence of rat sarcoma virus (RAS) mutations.

\section{Results}

RAS mutations occurred in $43 \%$ of the patients; $91 \%$ of these mutations were in K-ras. Seventy-five percent of these K-ras mutations were in codon 12, most commonly p.G12D. Codon 13 mutations occurred in $20 \%$ of tumors: all of these were p.G13D (100\%). The percentage of K-ras mutations occurrence was higher in young patients ( $\leqslant 50)$ compared with the older patients $(>50)(54.5 \%$ and $35 \%$, respectively). Similarly, the percentage of K-ras mutations occurrence was higher in the right-sided tumors compared with the left-sided tumors (57.1\% and 32.4\%, respectively). Patients' characteristics and clinicopathological features were not significantly associated with K-ras mutations.

Received 08/07/2020

Review began 08/08/2020 Review ended 08/14/2020 Published 08/24/2020

() Copyright 2020 Mulla et al. This is an open access article distributed under the terms of the Creative Commons Attribution License CC-BY 4.0., which permits unrestricted use, distribution, and reproduction in any medium, provided the original author and source are credited.

\section{Conclusions}

K-ras mutations are common among Saudi patients diagnosed with CRC in Madinah, especially pG12V and pG12D in codon 12. Further investigation would be required to establish correlation of K-ras mutations in larger cohorts.

\author{
Categories: Genetics, Pathology, Oncology \\ Keywords: colorectal cancer, crc, k-ras mutations, madinah, saudi arabia
}

\section{Introduction}

Colorectal cancer (CRC) is one of the leading types of cancer worldwide [1,2]. In Saudi Arabia, CRC is the most common cancer among males (11.2\%) and the third most common cancer among females $(8.8 \%)[3,4]$. CRC is very complicated at the molecular level which makes it reasonable to predict that patient's management is required to be stratified. To establish comprehensive patient stratification models, identification of targetable biomarkers which directs to therapeutic personalization will be crucial [5].

Kirsten-ras (K-ras) is an oncogene that is reported to be activated through mutations in $35 \%$ to $45 \%$ in patients with CRC [2]. Almost all of these K-ras mutations occur in codons 12 or 13 [6]. K-ras is the most common oncogene in the well-studied rat sarcoma virus (RAS) subfamily proteins because of its significant role in cancer [7]. Wild type or unmutated K-ras protein resides in the GDP-bound state on the plasma membrane in quiescent cells (i.e inactive). However, mutated K-ras will be in continuous stimulation which leads to the recruitment of specific guanine nucleotide exchange factors and significant conformational changes of the K-ras protein. These changes result in constitutive engagement and activation of K-ras effector pathways such as MAPK and PI3K-AKT signaling pathways. Subsequently, mutant K-ras proteins are 
driving tumor formation and progression [8]. Activated K-ras was shown to be associated with decreased responsiveness to targeted therapies such as anti-epidermal growth factor receptor (EGFR) antibodies in metastatic CRC as well as increased aggressiveness of CRC [9-12]. Therefore, characterizations of K-ras and other oncogenic drivers are increasingly useful to guide prognostication and molecularly stratified treatment options available to patients with CRC [13,14].

In this study, we aimed to investigate retrospectively the frequency of K-ras mutation and analyze its correlation with patients' characteristics and its association with clinicopathological features (i.e age, gender, clinical stage, anatomical site, histological subtype, degree of histological differentiation and metastatic site) in patients with CRC.

\section{Materials And Methods Study population}

A retrospective study was done at King Fahad Hospital, Madinah, Saudi Arabia. Histopathological and clinical data were obtained from the medical records of 51 patients with histologically proven colorectal adenocarcinoma. Tumor samples were obtained during surgery or biopsies carried out as part of routine care between 2016 and 2019. The histopathology reports were retrieved from the hospital's laboratory information system and were analyzed for data pertaining to age, gender, site of tumor, histologic type, tumor grade, nodal status and stage. The medical imaging records provided information on metastatic site. The RAS mutation results for all the cases were known, testing was done at Genekor Labs, Greece. All aspects of the study were approved by the local ethics committee.

\section{K-ras and N-ras mutation testing}

DNA extraction was carried out from formalin fixed paraffin embedded tissue. $6 \mu$ thick sections were taken on positively charged slides from a tumor block selected by the primary reporting histopathologist. These were sent to a referral laboratory (Genekor Lab, Greece). Macrodissection of the tissue was performed on direction of the histopathologist at the referring laboratory by marking the area on the template slide. Tumor cell content (TCC) $>75 \%$. DNA was extracted from the sample under investigation (QIAmp DNA FFPE tissue kit, QIAmp DSP DNA Mini kit).

A targeted resequencing assay (Ion AmpliSeq NGS Panel, Thermo Fisher Scientific, Indianapolis, IN) was used for mutation detection in exons 2, 3 and 4 of the K-ras and N-ras genes. Sequencing was carried out using the Next Generation Sequencing Platform Ion Gene Studio S5 Prime System (Thermo Fisher Scientific). The kit was used to detect mutation in p.G12S, p.G12R, p.G12C, p.G12D, p.G12A, p.G12C on Codons 12 and 13 of exon 2 . Exon 2 wild type cases were further analyzed for mutations in exons $3 \& 4$ of Kras genes and exons 2, $3 \& 4$ of $\mathrm{N}$-ras genes. The detection limit of the used method was $2 \%-5 \%$ of mutant allelic content, depending on the genomic region analyzed.

\section{Study design}

The overall frequency of the K-ras and N-ras mutations including type of mutation and affected codon were tabulated for all the cases with respect to age, gender, tumor site, tumor histologic type, American Joint Committee on Cancer (AJCC) stage and site of metastases. SPSS software (v20) was used to perform the statistical analysis. $\mathrm{P}<0.05$ was considered statistically significant.

\section{Results}

\section{1) Characteristics of patients}

Fifty-one patients with CRC were evaluated. The mean age was 60.2 years (range, 28-91). There was no difference in male to female ratio (i.e 1.04:1). In addition, all stages are included in the cohort. Most tumors were left sided (i.e $62.7 \%$ ). Adenocarcinoma accounts for $94 \%$ of the tumors and the site of metastasis was mainly liver. All the other tumor characteristics are shown in Table 1. 


\section{Cureus}

Gender

Male

Female

Stage

stage I

stage III

Site

Left

Right

Histology

Adenocarcinoma

Mucinous carcinoma

48

Tumor differentiation

\section{Moderate}

43

Poor

Well

Site of metastasis $(n=15)$

\section{liver, omentum}

Lung

lung and liver

mesentry, liver

Omentum

Retroperitoneum

\section{TABLE 1: Tumor characteristics of 51 patients with colorectal cancer}

\section{2) Characteristics of K-ras mutations}

Mutational analysis revealed RAS mutations in 43.1\% (22/51) of CRC patients (Table 2). Most mutations were in K-ras which accounts for $91 \%(20 / 22)$ while the remaining $9 \%(2 / 22)$ were detected in N-ras. In other words, K-ras mutations occurred in $39.2 \%$ of the patients (20 out of 51). Seventy-five percent of these K-ras mutations were in codon 12 ( 15 out of 20), most commonly p.G12D (53\% of codon 12 mutations). Codon 13 mutations occurred in $20 \%$ of tumors; all of these were p.G13D (100\%). 


\section{Cureus}

N

$\%$

RAS status

Wild type

Mutant type

29

56.9

22

Mutant type $\mathrm{N}=22$

K-ras

N-ras

K-ras $(n=20)$

Exon 2

Exon 4

K-ras $(n=20)$

Codon 12 (p.G12)

Codon 13 (p.G13D)

Codon 14 (p.A14)

K-ras Codon 12 (p.G12) $(n=15)$

p.G12A

p.G12C

p.G12D

p.G12S

p.G12V

$\mathrm{N}$-ras $(\mathrm{n}=2)$

Exon 2

Exon 3

$N$-ras $(n=2)$

p.G12D

p.Q61L
9.1

95

5

75

20

6.7

6.7

53.3

13.3

20

50

50

50

50

TABLE 2: Characteristics of rat sarcoma virus (RAS) mutations

3) Correlations between K-ras status (mutation vs wild type) and clinicopathological features

The percentage of K-ras mutations occurrence was higher in young patients (i.e $\leqslant 50$ ) compared with the older patients (>50) (54.5\% and 35\%, respectively). Similarly, the percentage of K-ras mutations occurrence was higher in the right-sided tumors compared with the left-sided tumors (57.1\% and $32.4 \%$, respectively)

(Table 3). On the other hand, the percentage of K-ras mutations occurrence was comparable between male and female ( $42.3 \%$ vs. $36 \%$ ) or between stages I to III and stage IV (38.2\% vs. $41.2 \%)$. There was no statistically significant association between the overall frequency of K-ras mutations and patients' characteristics and/or clinicopathological features (Table 3). 


\section{Cureus}

\begin{tabular}{|c|c|c|c|}
\hline & \multicolumn{2}{|c|}{ K-ras mutational status No (\%) } & \multirow{2}{*}{$P$ value } \\
\hline & Wild type & Mutated & \\
\hline \multicolumn{4}{|l|}{ Age } \\
\hline$\leq 50$ & $5(45.5)$ & $6(54.5)$ & \multirow{2}{*}{0.24} \\
\hline$>50$ & $26(65)$ & $14(35)$ & \\
\hline \multicolumn{4}{|l|}{ Gender } \\
\hline Male & $15(57.7)$ & $11(42.3)$ & \multirow{2}{*}{0.64} \\
\hline Female & 16 (64) & $9(36)$ & \\
\hline \multicolumn{4}{|l|}{ Stage } \\
\hline Stages I, II \& III & 21 (61.8) & $13(38.2)$ & \multirow{2}{*}{0.84} \\
\hline Stage IV & $10(58.8)$ & $7(41.2)$ & \\
\hline \multicolumn{4}{|l|}{ Site } \\
\hline Left & $25(67.6)$ & 12 (32.4) & \multirow{2}{*}{0.11} \\
\hline Right & $6(42.9)$ & $8(57.1)$ & \\
\hline \multicolumn{4}{|c|}{ Site of metastasis $(n=15)$} \\
\hline Liver & $9(75)$ & $3(25)$ & \multirow{2}{*}{0.17} \\
\hline Non-liver & $1(33.3)$ & $2(66.7)$ & \\
\hline
\end{tabular}

TABLE 3: Patients' characteristics and association between clinicopathological features and Kirsten-ras (K-ras) status

\section{Discussion}

Here, we are reporting the clinicopathological features of K-ras mutations in tumors derived from 51 patients diagnosed with colorectal cancer from 2016 to 2019 in Madinah city (western Saudi Arabia). We have found that $43 \%$ of the cohort carries RAS mutations with one-third of these mutations localized within codon 12 of K-ras. Characterizing the clinicopathological features of K-ras mutations in CRCs has carried increasing attention in recent years. One of the main reasons is due to the fact that CRC patients with K-ras mutations will not benefit from anti-EGFR antibodies [15].

Percentage of K-ras mutations, which are known to work as an activated oncogene, occurs in $35 \%-45 \%$ of CRC worldwide $[6,11,16]$. Results from these studies are in agreement with our observation that $\sim 40 \%$ of patients carry K-ras mutations. Few studies looked at the percentage of K-ras mutations in Saudi Arabia. For instance, a study reported that $32 \%$ of tumors (15/46) carry K-ras mutations [17]. Another study reported that $45 \%$ of tumors (97/221) carry K-ras mutations [18]. A third study reported that $56 \%$ of tumors $(84 / 150)$ carry K-ras mutations [19]. It is not clear why there is a wide range difference in the percentage of K-ras mutations in Saudi Arabia and there is a clear need for a national survey that considers the possible reasons for these variations.

One of the main objectives of this study is to look into K-ras status and its correlation with patients' characteristics as well as its association with clinicopathological features. We have found that the percentage of K-ras mutations occurrence was higher in young patients (i.e $\leqslant 50$ ) and in the right-sided tumors. However, we found no significant association between the overall frequency of K-ras mutations and patients' characteristics and/or clinicopathological features. In literature, conflicting results were documented regarding the association between K-ras mutations and patients' characteristics and/or clinicopathological features [17-21]. For example, while some studies showed that females are significantly more likely to have mutant K-ras than males [18,22], other studies showed the opposite (i.e males are significantly more likely to have mutant K-ras than females) [17,23]. Further studies will be required to determine whether mutant K-ras is significantly correlated with CRC patients' characteristics and clinicopathological features or not.

Delineating the pathogenetic importance of mutant K-ras in CRC is a successful step towards the control and management of this common type of cancer. In this study, 75\% of K-ras mutations were found in codons 12 
and $20 \%$ in codon 13 . A similar observation was reported by others in which they found $\sim 87 \%$ and $~ 14 \%$ of K-ras mutations in codons 12 and 13, respectively [17,19,24]. While lack of survival data is a limitation of our study, many studies showed that K-ras status did not significantly impact the clinical outcome and overall survival $[11,17,18]$. Due to the oncogenic nature of K-ras, we are currently following up on the patients included in this study for a longer period to correlate the survival data with K-ras mutations. We believe that it will be more informative to analyze survival data for each K-ras mutation in a large cohort. For example, results derived from studies that performed a comprehensive characterization of some commonly mutated oncogenes, such as p53 and anaplastic lymphoma kinase, concluded that prognostic significance of mutations is dependent on the region of the gene $[25,26]$.

\section{Conclusions}

In this small retrospective study, we showed that the rate of K-ras mutation in Saudi patients with CRC is $40 \%$ which is in agreement with most studies worldwide. K-ras mutation status did not significantly correlate with patients' characteristics and clinicopathological features. Lastly, our data has suggested that further investigation would be required to establish the pathogenetic importance of K-ras mutations in larger cohorts as well as to address the correlation and its prognostic value. Furthermore, longer follow up to address the survival are warranted.

\section{Additional Information \\ Disclosures}

Human subjects: Consent was obtained by all participants in this study. Ethics committee at King Fahad Hospital, Ministry of Health, Madinah, Saudi Arabia issued approval 300/275. Animal subjects: All authors have confirmed that this study did not involve animal subjects or tissue. Conflicts of interest: In compliance with the ICMJE uniform disclosure form, all authors declare the following: Payment/services info: All authors have declared that no financial support was received from any organization for the submitted work. Financial relationships: All authors have declared that they have no financial relationships at present or within the previous three years with any organizations that might have an interest in the submitted work. Other relationships: All authors have declared that there are no other relationships or activities that could appear to have influenced the submitted work.

\section{References}

1. Aziz MA, Allah-Bakhsh H: Colorectal cancer: a looming threat, opportunities, and challenges for the Saudi population and its healthcare system. Saudi J Gastroenterol. 2018, 24:196-197. 10.4103/sjg.SJG_164_18

2. Ahmed M: Colon cancer: a clinician's perspective in 2019. Gastroenterology Res. 2020, 13:1-10. 10.14740/gr1239

3. Al-Zahrani W, Al-Rawaji A, Al-Shahrani Z, Al-Madouj A: Cancer incidence report in Saudi for the year 2015. NHIC. 2018,

4. Al-Zahrani W, Al-Rawaji A, Al-Shahrani Z, Al-Madouj A: Cancer incidence report in Saudi for the year 2016. NHIC. 2019,

5. Fernandes E, Sores J, Cotton S, et al.: Esophageal, gastric and colorectal cancers: looking beyond classical serological biomarkers towards glycoproteomics-assisted precision oncology. Theranostics. 2020, 10:49034928. 10.7150/thno.42480

6. Arrington AK, Heinrich EL, Lee W, et al.: Prognostic and predictive roles of KRAS mutation in colorectal cancer. Int J Mol Sci. 2012, 13:12153-12168. 10.3390/ijms131012153

7. Li Z-N, Zhao L, Yu L-F, Wei M-J: BRAF and KRAS mutations in metastatic colorectal cancer: future perspectives for personalized therapy. Gastroenterol Rep. 2020, 8:192-205. 10.1093/gastro/goaa022

8. O'Bryan JP: Pharmacological targeting of RAS: recent success with direct inhibitors . Pharmacol Res. 2019, 139:503-511. 10.1016/j.phrs.2018.10.021

9. Kishiki T, Ohnishi H, Masaki T, et al.: Impact of genetic profiles on the efficacy of anti-EGFR antibodies in metastatic colorectal cancer with KRAS mutation. Oncol Rep. 2014, 32:57-64. 10.3892/or.2014.3179

10. Seo Y, Ishii Y, Ochiai H, et al.: Cetuximab-mediated ADCC activity is correlated with the cell surface expression level of EGFR but not with the KRAS/BRAF mutational status in colorectal cancer. Oncol Rep. 2014, 31:2115-2122. 10.3892/or.2014.3077

11. Dinu D, Dobre M, Panaitescu E, et al.: Prognostic significance of KRAS gene mutations in colorectal cancer-preliminary study. J Med Life. 2014, 7:581-587.

12. Van Cutsem E, Lenz HJ, Köhne $\mathrm{CH}$, et al.: Fluorouracil, leucovorin, and irinotecan plus cetuximab treatment and RAS mutations in colorectal cancer. J Clin Oncol. 2015, 33:692-700. 10.1200/jco.2014.59.4812

13. Kudryavtseva AV, Lipatova AV, Zaretsky AR, et al.: Important molecular genetic markers of colorectal cancer. Oncotarget. 2016, 7:53959-53983. 10.18632/oncotarget.9796

14. Sveen A, Kopetz S, Lothe RA: Biomarker-guided therapy for colorectal cancer: strength in complexity. Nat Rev Clin Oncol. 2020, 17:11-32. 10.1038/s41571-019-0241-1

15. Ibrahim EM, Zekri JM, Bin Sadiq BM: Cetuximab-based therapy for metastatic colorectal cancer: a metaanalysis of the effect of K-ras mutations. Int J Colorectal Dis. 2010, 25:713-721. 10.1007/s00384-010-0927-4

16. Domingo E, Ramamoorthy R, Oukrif D, et al.: Use of multivariate analysis to suggest a new molecular classification of colorectal cancer. The. J Pathol. 2013, 229:441-448. 10.1002/path.4139

17. Zekri J, Rizvi A, Al-Maghrabi J, bin Sadiq B: K-ras in colorectal cancer tumors from Saudi patients: frequency, clinco-pathological association and clinical outcome. Clin Colorectal Cancer. 2012, 5:22-27. $10.2174 / 1876820201205010022$ 


\section{Cureus}

18. Abulkair O, Alqahtani A, Gasmelseed A, Abdelhafiz N, Al Olayan A, Saadeddin A: KRAS mutational status and its clinical implications in Saudi colorectal cancer patients. PAJO. 2016, 9:12-18.

19. Zahrani A, Kandil M, Badar T, Abdelsalam M, Al-Faiar A, Ismail A: Clinico-pathological study of K-ras mutations in colorectal tumors in Saudi Arabia. Tumori J. 2014, 100:75-79. 10.1700/1430.15819

20. Bader T, Ismail A: Higher prevalence of KRAS mutations in colorectal cancer in Saudi Arabia: propensity for lung metastasis. Alexandria Med J. 2014, 50:203-209.

21. Zekri J, Al-Shehri A, Mahrous M, et al.: Mutations in codons 12 and 13 of K-ras exon 2 in colorectal tumors of Saudi Arabian patients: frequency, clincopathological associations, and clinical outcomes. Genet Mol Res. 2017, 16:10.4238/gmr16019369

22. Shen H, Yuan Y, Hu HG, et al.: Clinical significance of K-ras and BRAF mutations in Chinese colorectal cancer patients. World J Gastroenterol. 2011, 17:809-816. 10.3748/wjg.v17.i6.809

23. Zalis MG, Vieira FM, Zalcberg-Renault I, Bonamino MH, Ferreira CG, Oliveira S: KRAS mutation profile in colorectal cancer patients in Brazil: a cohort of 989 individuals. J Clin Oncol. 2009, 27: e15017-e15017. 10.1200/jco.2009.27.15 suppl.e15017

24. Karapetis CS, Khambata-Ford S, Jonker DJ, et al.: K-ras mutations and benefit from cetuximab in advanced colorectal cancer. N Engl J Med. 2008, 359:1757-1765. 10.1056/NEJMoa0804385

25. Powell B, Soong R, Iacopetta B, Seshadri R, Smith DR: Prognostic significance of mutations to different structural and functional regions of the p53 gene in breast cancer. Clin Cancer Res. 2000, 6:443-451.

26. Bresler SC, Weiser DA, Huwe PJ, et al.: ALK mutations confer differential oncogenic activation and sensitivity to ALK inhibition therapy in neuroblastoma. Cancer Cell. 2014, 26:682-694. 10.1016/i.ccell.2014.09.019 